\title{
Mathematical Model of Influence of Geometric Parameters on Wave Gear Teeth Wear
}

\author{
I. Ye. Lyuminarskiy ${ }^{1}, S . Y e$. Lyuminarskiy ${ }^{1}$, and Ye. S. Lyuminarskaya ${ }^{1}{ }^{*}$ \\ ${ }^{1}$ Bauman Moscow State Technical University, Block 1, Bldg. 5, 2nd Baumanskaya St., Moscow, the \\ Russian Federation
}

\begin{abstract}
The object of the research is a wave gear with a cam wave generator. One of the requirements for wave gears is to increase the wear resistance of the teeth flanks. A mathematical model that makes it possible to assess the influence of the geometrical parameters of the gear on the teeth wear is proposed in this work. To assess the specified effect, the maximum and average wear coefficients are introduced. To calculate these coefficients, a spatial mathematical model of a wave gear is used, taking into account the volumetric and contact deformations of gear wheels and a flexible bearing. Using the developed technique, the dependences of the wear coefficients on the radial deformation and the length of the flexible gear were obtained. The given dependences increase the knowledge concerning the influence of geometric parameters on the wave gear teeth wear.
\end{abstract}

\section{Introduction}

One of the requirements for wave gears (WG) is to increase the wear resistance of the teeth [1-3]. The skew of the generatrix of the flexible gear leads to the edged interaction of the teeth of the flexible and rigid gears. Edged contact, uneven distribution of forces among the teeth and high sliding motion of the teeth lead to long breaking-in of the teeth and low wear resistance. Increased wear increases kinematic error and gear backlash. Reducing the intensity of wear of the side surfaces will reduce the breaking-in time of the teeth and make it possible to change quality indicators during operation. Usually, in engineering calculations, the assessment of the wear of the teeth flanks is not performed, and the wear resistance of the teeth is checked experimentally. Therefore, the development of methods for the assessment of the influence of geometric parameters on the wave gear teeth wear is relevant. Such methods will make it possible to increase the wear resistance of the teeth by changing the gear geometric parameters at the design stage.

\section{Statement of problem}

The works $[4,5]$ are dedicated to the issues of wear of sliding surfaces. The theoretical definition of the wear of cylindrical gears with fixed axles of the wheels is considered in

\footnotetext{
* Corresponding author: katjstas@mail.ru
} 
Ye.P. Kovalev's dissertation [6]. The authors show that the wear of sliding surfaces depends on contact stresses, materials of sliding surfaces, lubricant and ambient temperature. In this work, to determine the influence of geometric parameters on the wear of the teeth flanks, it is proposed to take into account the change in contact stresses only. To assess this effect, it is necessary to develop criteria for wear assessment and a methodology for their calculation, which should take into account the spatial nature of the elastic interaction of the gear elements.

The purpose of this work is to develop a mathematical model for the assessment of the influence of geometric parameters on the wear of the flanks of the wave gear teeth and to study the effect of radial deformation and the length of a flexible gear on the teeth wear intensity.

\section{Mathematical model}

A wave gear with a cam wave generator and a fixed rigid gear is shown in Fig. 1. To determine the contact stresses $\sigma_{H}$ on the teeth flanks, the elastic interaction of the WG elements is calculated. The calculation takes into account the elastic deformations of the flexible (FG) and rigid (RG) gears, the outer ring of the flexible bearing (ORFB), contact deformations in the flexible bearing (FB), and displacement of the WG elements as rigid bodies. To determine the forces of interaction of transmission elements, the spatial model of WG is used [7, 8].

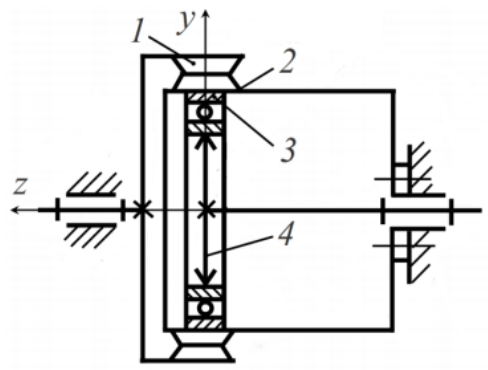

Fig. 1. WG Scheme: 1 - rigid gear; 2 - flexible gear; 3 - flexible bearing; 4 - cam

The resolving system is compiled using the Bubnov-Galerkin method. The forces of interaction of WG elements distributed over the flanks are replaced by surface forces with piecewise linear distribution. For this purpose, interacting flanks are divided into rectangular subareas. A dimensionless Courant function $\varphi_{j}$ [9], which represents a hexagonal pyramid with unit height, is assigned to each grid node. The following functions are used as basic ones: $u_{j}=\left(\frac{3}{s_{j}}\right) \varphi_{j}$, where $S_{i}$ - base area of the pyramid. The basic functions $u_{j}$ are unit volume hexagonal pyramids.

Thus, the intensity of the distributed force of interaction between two surfaces is represented by the following linear combination of basic functions:

$$
p=\sum\left(F_{j} u_{j}\right)
$$

where coefficients $F_{j}$ have the dimension of force and are determined as a result of the calculation of the resolving system of equations. Hereinafter, coefficients $F_{j}$ are called the forces of interaction of surfaces.

The system of resolving equations has the following form: 


$$
\left\{\begin{array}{l}
\left(\begin{array}{ll}
\boldsymbol{D} & \boldsymbol{G} \\
\boldsymbol{G} & \boldsymbol{C}
\end{array}\right) \cdot\left(\begin{array}{c}
\boldsymbol{F} \\
a
\end{array}\right)=\left(\begin{array}{c}
\widetilde{\boldsymbol{\delta}}-\widetilde{\boldsymbol{\delta}}_{\mathbf{0}} \\
\boldsymbol{B}
\end{array}\right) \\
\tilde{\delta}_{j} \geq 0, j=1, \ldots, N \\
F_{j} \geq 0, j=1, \ldots, N \\
\tilde{\delta}_{j} \cdot F_{j}=0, j=1, \ldots, N
\end{array}\right.
$$

where $\boldsymbol{F}=\left\|\boldsymbol{F}^{(1) T}, \boldsymbol{F}^{(2) T}, \boldsymbol{F}^{(3) T}\right\|^{T}-$ vector of forces of interaction of conjugated surfaces; $a=\left\|a^{(1) T}, a^{(2) T}, a^{(3) T}\right\|^{T}-$ displacement vector of gear elements as rigid bodies; $\widetilde{\boldsymbol{\delta}}, \widetilde{\boldsymbol{\delta}}_{\mathbf{0}}$ - vector of reduced gaps at node points between surfaces in deformed and nondeformed states of gear elements; $\boldsymbol{B}$ - vector of external forces and moments; $\boldsymbol{D}$ - nodal compliance matrix; $\boldsymbol{G}$ - matrix connecting reduced clearance increments $\tilde{\delta}_{j}$ with displacement vector $a$.

Herein, $\boldsymbol{F}^{(1)}, \boldsymbol{F}^{(2)}, \boldsymbol{F}^{(3)}$ - vectors of interaction forces of RG and FG, FG and ORFB, ORFB and flexible bearing balls, respectively; $a^{(1)}=\left\|\Delta x^{(1)}, \Delta y^{(1)}\right\|^{T}, a^{(2)}=$ $\left\|\Delta x^{(2)}, \Delta y^{(2)}, \Delta \varphi_{z}^{(2)}\right\|^{T}, a^{(3)}=\left\|\Delta x^{(3)}, \Delta y^{(3)}, \Delta \varphi_{x}^{(3)}, \Delta \varphi_{y}^{(3)}\right\|^{T}$ - displacement vectors of $\mathrm{RG}$, FG and ORFB, respectively.

The system of resolving equations (1) is a set of canonical equations of the method of forces, equilibrium equations of RG, FG, ORFB, and inequalities expressing the one-sided nature of the interaction of gear elements. Influence factors are determined using linear shell theory and the finite element method. The calculation procedure is described in more detail in [7].

The intensity of wear at a certain point on the tooth flank depends on the value of the contact stresses $\sigma_{H}$, material of sliding surfaces, ambient pressure $P_{c}$, maximum contact temperature $T_{c}$ and other factors [4-6]:

$$
J=\exp (k) \cdot\left(\frac{\sigma_{H}}{H B}\right)^{\beta_{1}} \cdot\left(\frac{P_{c}}{P_{c r}}\right)^{\beta_{2}} \cdot\left(\frac{T_{c}}{T_{c r}}\right)^{\beta_{3}},
$$

where $\sigma_{H}$ - normal contact stresses; $H B$ - Brinell hardness of contact surfaces; $P_{c r}-$ saturated vapor pressure at critical temperature; $T_{c r}$ - critical temperature for the lubricant used; $k, \beta_{1}, \beta_{2}, \beta_{3}$ coefficients depending on gear materials, lubricant and ambient temperature.

The gear geometrical parameters have little effect on the pressure and temperature in the contact area. Therefore, the article proposes to take into account only the effect of contact stresses on the wear rate:

$$
J=a \cdot \sigma_{H}^{\beta_{1}},
$$

where $a$-constant coefficient; $\beta_{1}$ - coefficient, the value of which in the article is taken to be 1 .

When determining contact stresses, the working flanks of the FG teeth are divided into rectangular areas. The amount of wear of the FG $i^{\text {th }}$ area when the cam is rotated by one revolution is calculated by the following formula:

$$
h_{i}=\sum_{j=1}^{z_{g}} a \cdot \sigma_{H i j} \beta_{1} \cdot\left|\frac{\Delta W_{i, j}}{\cos \alpha_{i}}\right|,
$$

where $\sigma_{H i j},\left|\frac{\Delta W_{i, j}}{\cos \alpha_{i}}\right|$ - average contact stress and friction path of the $i^{\text {th }}$ area of the $j^{\text {th }}$ tooth of the FG when the cam is rotated at angle $\Delta \varphi_{H}=\frac{2 \pi}{z_{g}} ; \Delta W_{i, j}$ - radial displacement of the center of gravity of the area under consideration; $\alpha_{i}-$ angle of FG tooth outline at the center of gravity of the area under consideration; $z_{g}$ - number of FG teeth. 
To assess the influence of geometric parameters on the wear of the teeth flanks, it is proposed in the article to use the following coefficients:

1) wear coefficient at a given point on the flank

$$
\xi_{i}=\frac{h_{i}}{a}=\sum_{j=1}^{z g} \sigma_{H i j} \beta_{1} \cdot\left|\frac{\Delta W_{i, j}}{\cos \alpha_{i}}\right|,
$$

2) average wear coefficient of the teeth flank

$$
\xi_{a v}=\frac{1}{N} \sum_{i=1}^{N} \xi_{i}
$$

3) wear coefficient of the teeth flank

$$
\xi=\max _{i}\left(\xi_{i}\right) .
$$

Herein, $N$ - number of areas into which the tooth flank is divided; $i$ - area number; $j-$ FG tooth number.

The applied coefficients are proportional to the wear of the flanks per one cam revolution.

\section{Research results}

For the desktop research, a wave gear with a cam wave generator and the following parameters was chosen: RG teeth number $z_{b}=202$, FG teeth number $z_{g}=200$, module $m$ $=0.8 \mathrm{~mm}$, length of tooth $b_{w}=32 \mathrm{~mm}$, length of FG $L=160 \mathrm{~mm}$, outer diameter of flexible bearing $D=160 \mathrm{~mm}, \mathrm{FG}$ maximum deformation $w_{0}=1,1 \mathrm{~m}$. The other dimensions of the wave gear were taken in accordance with GOST 30078.2-93, the dimensions of flexible bearings - in accordance with GOST 2379-78 and the recommendations given in [10]. The desktop research was carried out at the moment of resistance on the output shaft $M_{C}=400$ $\mathrm{N} \cdot \mathrm{m}$. The standard flexible bearing is located in the middle of the FG ring gear.

Figure 2 shows the dependences of the wear coefficients $\xi_{a v}$ and $\xi$ on the maximum radial deformation of the flexible gear $w_{0}$. The value of the teeth wear coefficient $\xi$ is $2 \ldots 4$ times higher than the value of the averaged wear coefficient $\xi_{a v}$, which indicates the uneven wear of the teeth flanks. The increase $w_{0}$ from $0.8 \mathrm{~mm}$ to $0.86 \mathrm{~mm}$ results in the significant increase in the width of the contact patterns of the teeth and, consequently, to the decrease in the coefficient $\xi_{a v}$. The increase in $\xi_{a v}$ with $w_{0}>0.86 \mathrm{~mm}$ is caused by the removal of the contact area of the teeth from the major axis of the wave generator and the increase in the skew of the FG generatrix. The teeth wear area does not exceed $50 \%$ of the flank areas.

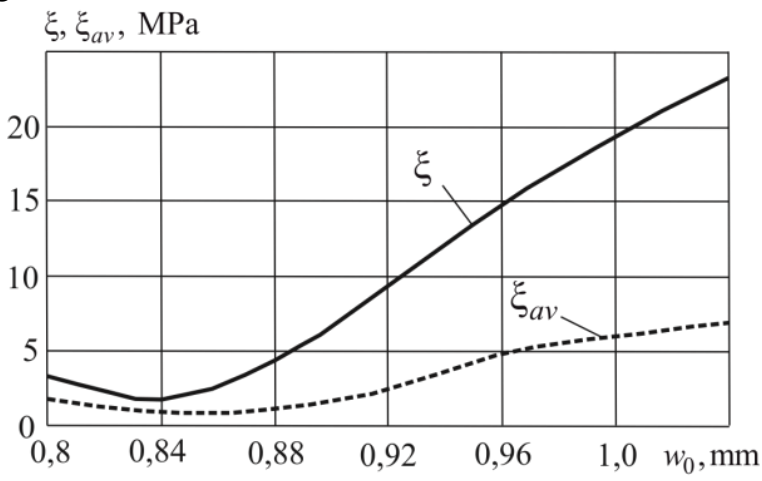

Fig. 2. Dependence of wear coefficients on FG initial deformation 
Figure 3 shows the dependences of the wear coefficients $\xi_{a v}$ and $\xi$ on the length of the flexible gear $L$. With the increase in the FG length, the width of the contact patterns of the teeth increases and, consequently, the contact stresses decrease. The change of the flexible gear length from $80 \mathrm{~mm}$ to $160 \mathrm{~mm}$ results in a decrease in the wear coefficient $\xi 4.5$-fold and the average coefficient $\xi_{a v}-6$-fold. Thereby, the wear area increases approximately twofold.

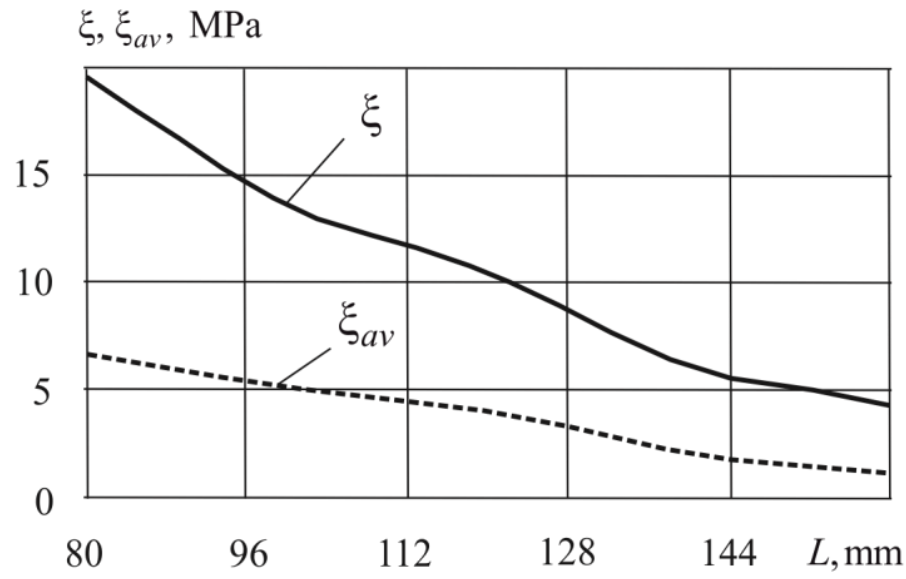

Fig. 3. Dependence of wear coefficients on FG length

\section{Conclusion}

1. A method is proposed to assess the influence of the WG geometric parameters on the wear rate of the teeth flanks.

2. The intensity of wear of the teeth flanks can be significantly reduced by changing the gear geometric parameters.

3 . For the gear under consideration, a 2-fold increase in the FG length reduces the wear rate of the flanks approximately 4 -fold, and a decrease in the FG maximum deformation by $4 \%$ leads to a decrease in the wear rate by $25 \%$.

\section{References}

1. Ginzburg, Ye.P. Wave Gear. - L., Mashinostroyeniye, 1969. - 200 p.

2. Volkov, D.P., Kraynev, A.F. Wave Gears. Kiev. Tekhnika. 1976. - 224 p.

3. Poletuchiy, A.I. Theory and Design of High Performance Wave Gear Mechanisms. Kharkov, Kharkov Aviation Institute Named after N.Ye. Zhukovskiy, 2005. - 675 p.

4. Kragelskiy, I.V. Frictional Interaction of Solids. Friction and Wear, 1980, V. 1, No. 1, P. 12-29.

5. Drozdov, Yu.N., Pavlov, V.G., Puchkov, V.N. Friction and Wear in Extreme Conditions: Handbook. - M.: Mashinostroyeniye. - 1986, 223 p.

6. Kovalev, Ye.P. Development of Prediction Technique for Resource of Novikov Gears in Vacuum. Dissertation of Candidate of Technical Science. Moscow, 1983. 131 p.

7. Lyuminarskiy, S.Ye., Lyuminarskiy, I.Ye. Mathematical Model of Wave Gear with Disk Wave Generator. Mechanical Engineering and Engineering Education, 2011, No. 2, P. 45-52. 
8. Lyuminarskiy, I.Ye., Lyuminarskiy, S.Ye. Mathematical Modeling of Limiting Regime of Wave Gear Operation // Proceedings of Universities. Mechanical Engineering Series. 2015. No. 12. P. 17-26.

9. Marchuk, G.I. Computational Mathematics Methods. St. Petersburg, Lan, 2011. 608 p.

10. Ivanov, M.N. Wave Gears. Textbook for University Students. Moscow, Vysshaya Shkola, 1981. 184 p. 